\title{
Evidence for a bound H-dibaryon using lattice QCD
}

\author{
William Detmold ${ }^{1}$ \\ Department of Physics, College of William \& Mary, Williamsburg, VA 23187, USA \\ Jefferson Lab, 12000 Jefferson Avenue, Newport News VA 23606, USA
}

\begin{abstract}
The $H$-dibaryon, a $J=0$ state with the valence quark content $u d s u d s$, has long been hypothesised to exist because of the attractive nature of colour magnetic gluon exchange in the flavour- singlet channel. Using lattice QCD the NPLQCD collaboration have investigated this system and evidence is presented for the existence of a stable $H$-dibaryon, albeit at a quark mass somewhat larger than that in nature. This calculation is reviewed and combined with subsequent calculations by the HALQCD collaboration at the SU(3) flavour symmetric point to identify bounds on the $H$-dibaryon mass at the physical quark masses.
\end{abstract}

Keywords: Lattice QCD

PACS: $12.38 \mathrm{Gc}$

\section{THE $H$-DIBARYON}

In the late 1970s, Jaffe [1] predicted the existence of a relatively deeply bound system with the quantum numbers of $\Lambda \Lambda$ (called the $H$-dibaryon) using the MIT bag-model. This finding started an ongoing search for such a system, both experimentally and also with alternate theoretical tools. Subsequent studies in the context of various hadronic models have predicted that a very wide range of masses for the $H$, anywhere from $\sim 1 \mathrm{GeV}$ (where the state would be absolutely stable) to well above the $\Lambda \Lambda$ threshold $\left(2 M_{\Lambda}=2.23 \mathrm{GeV}\right)$ where strong decays become possible, see [2] for a recent review. Experimental studies of doubly-strange hypernuclei and results from heavy ion collisions at RHIC [3] suggest that the $H$ is likely not bound ${ }^{2}$. Recent experiments at KEK suggest there is a resonance near threshold in the H-dibaryon channel [5]. These results and a range of theoretical investigations are reviewed in Refs. [6, 2, 7, 8].

\section{QCD CALCULATION}

Quantum chromodynamics (QCD), the theory describing the dynamics of quarks and gluons, is now well established and, in combination with the electroweak interactions, underlies all of nuclear physics, from the hadronic mass spectrum to stellar nucleosynthesis. Nevertheless, QCD has had only limited impact in nuclear physics because of the computational challenges in applying it in the low energy regime. Fortunately, QCD, through is numerical implementation as lattice QCD (QCD formulated on a discrete Euclidean spacetime lattice), is entering an era in which precise predictions for hadronic quantities with quantifiable errors are being made. One such prediction that has been made recently is that a stable $H$-dibaryon exists [9] at unphysically heavy up and down quark masses (corresponding to a pion mass $m_{\pi} \sim 389 \mathrm{MeV}$ ) in the isospin limit in the absence of electroweak interactions.

QCD studies of the $H$-dibaryon have a long history, with a range of exploratory quenched calculations $[10,11,12$, $13,14,15]$ finding inconclusive results. Recent algorithmic improvements and the growth of computational resources that are available have improved the situation and high precision QCD calculations of systems with the quantum numbers of the $H$ can now be performed with control over many of the dominant systematics.

As discussed in detail in Refs. [16, 17, 18, 9], the study of two-hadron systems in QCD is based on determinations of the dependence of two-hadron energy levels on the finite volume used in the lattice calculation. For scattering states, the phase shift, $\delta(q)$, at a particular energy can be extracted from the shift of the two body energy from that of two

\footnotetext{
1 Presented on behalf of the NPLQCD collaboration

${ }^{2}$ It has been suggested that if the $H$ state is tightly bound [4] or very weakly bound, the experimental limits can be evaded.
} 
non-interacting hadrons, $\Delta E=E_{2 H}-2 E_{H} \equiv 2 \sqrt{q^{2}+E_{H}^{2}}$, through the eigenvalue equation

$$
q \cot \delta(q)=\frac{1}{\pi L} S\left(q^{2}\left(\frac{L}{2 \pi}\right)^{2}\right), \quad \text { where } \quad S(x)=\lim _{\Lambda \rightarrow \infty} \sum_{\mathbf{j}}^{|\mathbf{j}|<\Lambda} \frac{1}{|\mathbf{j}|^{2}-x}-4 \pi \Lambda .
$$

In the above expression, the summation is over integer triplets $\mathbf{j}$ and we assume only $s$-wave interactions. This method has been used extensively in recent years to study a number of meson-meson, meson-baryon and baryon-baryon systems, see Ref. [19] for a review.

The natural extension of this formalism to negative values of $q^{2}$ allows for the identification of bound states. A bound state, which corresponds to a pole in the $\mathrm{S}$ matrix in the case of a single scattering channel ${ }^{3}$, is signaled by the phase shift satisfying $\cot \delta(q)=i$. Writing $q=i \kappa$ for states that are negatively shifted in energy in the lattice volume, the volume dependence of the binding momentum in the large volume limit follows from eq. (1) in a straightforward manner and is of the form [18]

$$
\kappa=\gamma+\frac{g}{L}\left(e^{-\gamma L}+\sqrt{2} e^{-\sqrt{2} \gamma L}\right)+\ldots,
$$

where $\gamma$ is the infinite-volume value of the binding momentum. In the above, we have assumed that $\gamma \ll m_{\pi}$, and $g$ is an undetermined parameter.

Ref. [9] studies the energy of the ground state of the system with the quantum numbers of the $H$ using four ensembles of gauge configurations generated with $2+1$ flavours of light quarks using an anisotropic clover discretisation [20, 21]. These correspond to a spatial(temporal) lattice spacing of $0.125 \mathrm{fm}(0.036 \mathrm{fm})$, a pion mass of $m_{\pi} \sim 389 \mathrm{MeV}$ and lattice volumes of side lengths $L=2.0,2.5,3.0$ and $3.9 \mathrm{fm}$. By examining the volume dependence of single particle energies and dispersion relations, the smallest two volumes were shown to be contaminated by systematic effects. However the ensembles with $L=3.0$ and $3.9 \mathrm{fm}$ were found to be sufficiently large for studies of two hadron dynamics. Negatively shifted $H$-dibaryon energies were seen on both volumes and, using these calculations, an extrapolation was performed using Eq. (2). The resulting infinite volume binding was found to be $B_{H}=16.6 \pm 2.1 \pm 4.6 \mathrm{MeV}$ at $m_{\pi} \sim 389$ $\mathrm{MeV}$ where the first uncertainty is statistical and the second includes estimates of remaining systematic effects.

\section{QUARK MASS DEPENDENCE}

Subsequent to the calculation discussed above, results showing a bound $H$ were presented in the SU(3)-flavour symmetric limit of QCD by the Inoue et al. [22, 23] and in quenched QCD by Luo et al. [24]. By incorporating the additional information contained in these calculations, an exploration of the mass dependence of the $H$ state is possible and tentative extrapolations to the physical quark mass can be made. Such a study was first performed in Ref. [25]where simple linear and quadratic extrapolations in the pion mass were used because of the uncertainties that arise if the state is an extended deuteron-like object at lighter quark masses. More complicated forms of the extrapolation involving chiral loops coupling to a compact object were considered in Ref. [26] (the assumption of a compact state requires careful investigation as the binding energy approaches threshold). These extrapolations are summarised in Fig. 1 and found binding energies and $12 \pm 7 \mathrm{MeV}$ (quadratic), $5 \pm 8 \mathrm{MeV}$ (linear) and $-13 \pm 14 \mathrm{MeV}$ (chiral), respectively. Clearly the existence of the $H$ at the physical point is currently ambiguous even in the isospin limit of QCD with electromagnetism turned off. Further QCD calculations at lighter quark masses will significantly improve our knowledge of this system. Such investigations are ongoing and will provide interesting input into future experimental investigations.

\section{ACKNOWLEDGMENTS}

I am grateful to my colleagues in the NPLQCD collaboration. This work was supported in part by the US Department of energy under grants DE-AC05-06OR23177 (JSA) and DE-FG02-04ER41302 and OJI grant DE-SC0001784 and by the Jeffress Memorial Trust, grant J-968

\footnotetext{
${ }^{3}$ Away from the SU(3) flavour symmetric point, the $H$-dibaryon should be discussed in the context of the $\Lambda \Lambda-\Xi N-\Sigma \Sigma$ coupled channels system However at the quark masses used in Ref. [9], the gaps between the three thresholds are significant and the coupled channel aspects are neglected.
} 


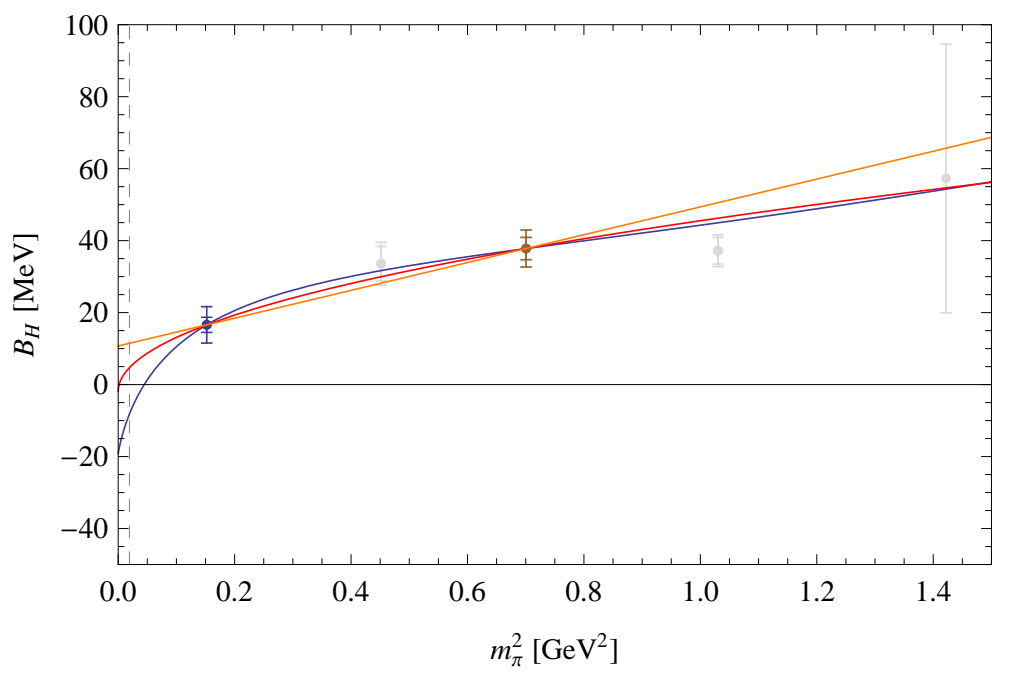

FIGURE 1. Simple extrapolations of recent lattice QCD calculations of the $H$-dibaryon binding energy, $B_{H}$ to the physical quark mass (dashed vertical line). The red(orange) curves correspond to extrapolations linear(quadratic) in the pion mass [25] while the blue curve uses the chiral non-analytic form proposed in Ref. [26] which assumes a tightly bound (compact) system. The data shown correspond to results presented in Refs. $[9,22,24]$, however, only the most physically relevant points (blue=NPLQCD, brown=HALQCD) are used in the extrapolations, as discussed in Ref. [25].

\section{REFERENCES}

1. R. L. Jaffe, Phys. Rev. Lett. 38, 195 (1977); 38, 617 (1977)(E).

2. T. Sakai, K. Shimizu and K. Yazaki, Prog. Theor. Phys. Suppl. 137, 121 (2000).

3. A. L. Trattner, PhD Thesis, LBL, UMI-32-54109 (2006).

4. G. R. Farrar, G. Zaharijas, Phys. Rev. D70, 014008 (2004). [hep-ph/0308137].

5. C. J. Yoon et al., Phys. Rev. C 75, 022201 (2007).

6. K. Yamamoto et al., Phys. Lett. B 478 (2000) 401.

7. P. J. Mulders and A. W. Thomas, J. Phys. G 9, 1159 (1983).

8. S. Bashinsky, R. L. Jaffe, Nucl. Phys. A625, 167-191 (1997). [hep-ph/9705407].

9. S. R. Beane et al. [ NPLQCD Collaboration ], Phys. Rev. Lett. 106, 162001 (2011).

10. P. B. Mackenzie and H. B. Thacker, Phys. Rev. Lett. 55, 2539 (1985).

11. Y. Iwasaki, T. Yoshie and Y. Tsuboi, Phys. Rev. Lett. 60, 1371 (1988).

12. A. Pochinsky, J. W. Negele and B. Scarlet, Nucl. Phys. Proc. Suppl. 73, 255 (1999).

13. I. Wetzorke, F. Karsch and E. Laermann, Nucl. Phys. Proc. Suppl. 83, 218 (2000).

14. I. Wetzorke and F. Karsch, Nucl. Phys. Proc. Suppl. 119, 278 (2003).

15. Z. H. Luo, M. Loan and X. Q. Luo, Mod. Phys. Lett. A 22, 591 (2007).

16. M. Lüscher, Commun. Math. Phys. 105, 153 (1986).

17. M. Lüscher, Nucl. Phys. B 354, 531 (1991).

18. S. R. Beane et al, [NPLQCD], Phys. Lett. B 585, 106 (2004).

19. S. R. Beane, W. Detmold, K. Orginos, M. J. Savage, Prog. Part. Nucl. Phys. 66, 1-40 (2011).

20. H. W. Lin et al. [HS], Phys. Rev. D 79, 034502 (2009).

21. R. G. Edwards, B. Joo and H. W. Lin, Phys. Rev. D 78, 054501 (2008).

22. T. Inoue et al. [ HAL QCD Collaboration ], Phys. Rev. Lett. 106, 162002 (2011).

23. T. Inoue, these proceeedings.

24. Z. -H. Luo, M. Loan, Q. -G. Lin, Y. Liu, arXiv:1106.1945 [hep-lat].

25. S. R. Beane, et al., arXiv:1103.2821.

26. P. E. Shanahan, A. W. Thomas, R. D. Young, arXiv:1106.2851. 